\title{
System of the Monitoring the Aircraft Construction in the Tests Process
}

\author{
A. P. Kozlov \\ National Aviation University \\ Kyiv, Ukraine \\ ap_kozlov@ukr.net
}

\author{
A. S. Yurchenko \\ National Aviation University \\ Kyiv, Ukraine \\ ayurchenko@yahoo.com
}

\begin{abstract}
The necessity of the organization and implementation of continuous monitoring of deformations in the dements of the aircraft construction for the early detection of critical values is shown. The short review of existing methods and devices for measuring micro-movements is given. Variants of designs of micro-movements sensors are considered and a block diagram of a measuring instrument, which was built on the basis of a capacitive transducer with an "open" field, is given. The functioning of the system of non-contact measurement of deformation elements of the aircraft structure is considered.
\end{abstract}

\section{Keywords-aircraft design; sensor; destruction of thestructure}

\section{INTRODUCTION}

The operational life of the aircraft largely depends on the strength and rigidity of its design. In the process of developing of new aircraft these settings are checked by compulsory set of tests. This complex consists of static, dynamic and flight tests of individual elements and components design and aircraft in general. The latter is crucial for assessing the strength of the design. During static tests the residual strain is determined after removing the pilot load, distribution of efforts on certain elements of design, meaning of the maximum load that can withstand the design to destruction. Dynamic tests are conducted to detect fatigue deformation. This basically refers to various bolt, rivet, welded and other connections. Flying tests are designed to verify the results of ground tests in real conditions. Test results largely depend on the methods and means of measurement parameters controlled [1].

\section{ANALYSIS OF PROBLEM}

During operation of aircraft design, its units and some parts are under various loads. Moreover, for some parts of the aircraft and its units may be the most dangerous forces acting in flight, for others - during take-off and landing. The nature of these forces can be different Permanent nature - from loading of aircraft, lift forces, variable nature - from the actions of aerodynamic forces episodic - from shock in case of emergency situations. As a result of these forces the fatigue strains are accumulated, which are characterized by a gradual increase in irreversible changes in the structure of the material and the appearance of cracks with subsequent formation macrocrack backbone, which leads to the destruction of the structure.
It is known that the elastic deformation structure recovers its shape after the termination of external forces, and the appearance of plastic deformation signals the beginning of the destruction process of design. Most parts and main elements of aircraft construction are made from different metals, which have different characteristics ductility, hardness and strength.

The total dependency deformation of elastic metals from loading (Hooke's law), used in creating the basic units of aircraft elements, is shown in Fig. 1. As seen from this graph, plot $O A$ corresponds to the elastic deformation and site $A B$ describes the occurrence of plastic deformation. Most design elements have a high degree of rigidity and strength of the material and therefore very low degree of plasticity. Any occurrence of plastic deformation is quite dangerous, so control of the appearance of plastic deformations of basic elements is extremely important to prevent the destruction of the aircraft design.

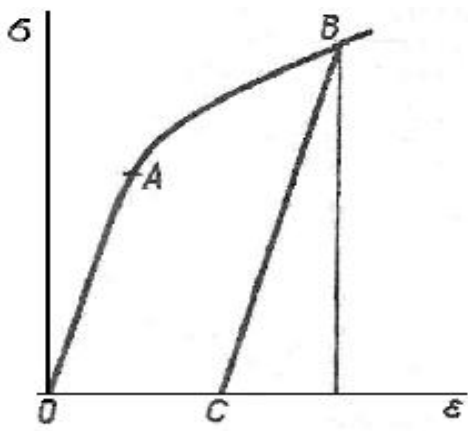

Fig. 1. Graph of elastic deformation of metals: $\sigma$ is the stress, $\varepsilon$ is the deformation.

The maintenance of aircraft design is in accordance with the regulations, which is provided after definite time of the operation by the repair or replacement of individual components and construction elements according to the requirements of operational documentation. Terms, composition and procedure of routine maintenance is based on the results before operational tests. During operation between the regulation control the monitoring of aircraft design is only sporadically. This is done in cases where the search of microcracks is performed after the rough, unsuccessful landing, rolling on the runway, and so on. If the aircraft exceeded its defined rules lifetime, it is removed from the exploitation and 
write off, despite the fact that it may become even allow further operation. However, there are cases where the destruction of any element of construction was still ending the resource, which is provided the regulations, it often ends by accident. So, it points to a clear need of continuous and constant control of the aircraft design for the entire operation. Such control will reduce the likelihood of accidental destruction of structures during the operation, and will extend the life of the aircraft in case of a full-time status at the end of the procedural deadline. This is useful both in terms of safety and cost.

For the organization and implementation of continuous control of PC designs it is necessary to decide the following:

1) to determine the structural elements, which is most likely to have origin of the devastating deformities;

2) to develop a set of measurement tools and methods of their use.

It is difficult enough in the short term that it can not be resolved. However, it is possible the ways of solutions can be outlined. Analysis of factors of accidents and flight events shows that the largest number of damage occurs in the joints of the wing with fuselage, in fixing engines on the wings and in the connecting element structures that attach the chassis. Thus, the placement in these areas the strain sensors with transmission of received information to the computer for further processing is the most appropriate solution for its appointed task. Some of the most common design of interelement bindings were considered for the selection of the measuring devices and their placement on the design elements of structure aircraft. The design of contour connecting of the wing attachment is shown on Fig. 2 [1].

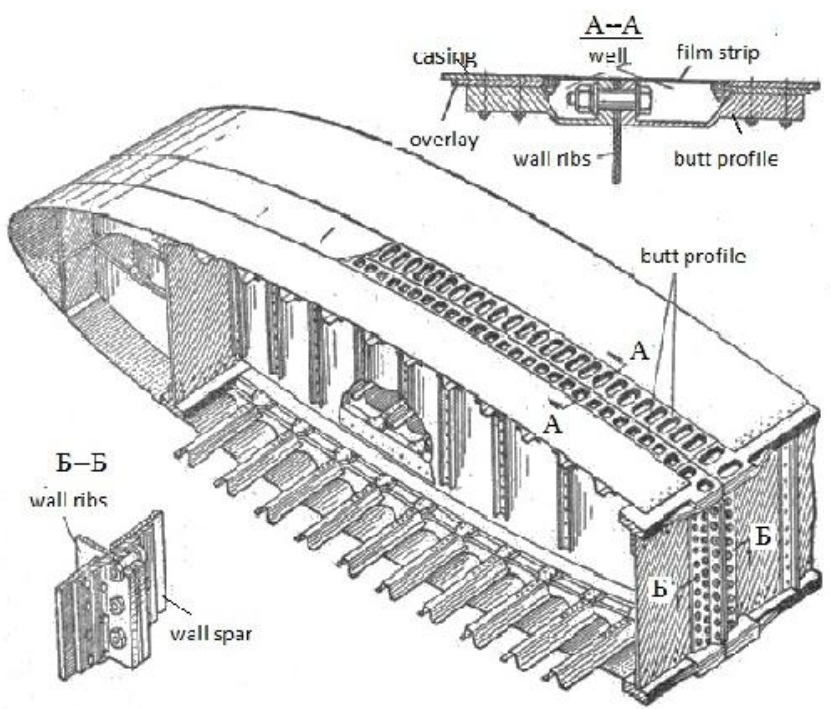

Fig. 2. The design of the mounting contour connecting wings, which is carried out by butt profiles.

Analysis of the possible burden of these elements suggests that the most likely area of dangerous strains is an abutting profiles in the field of fine section.

The construction junction is considered on Fig. 3, using the butt profile for interelement connection.
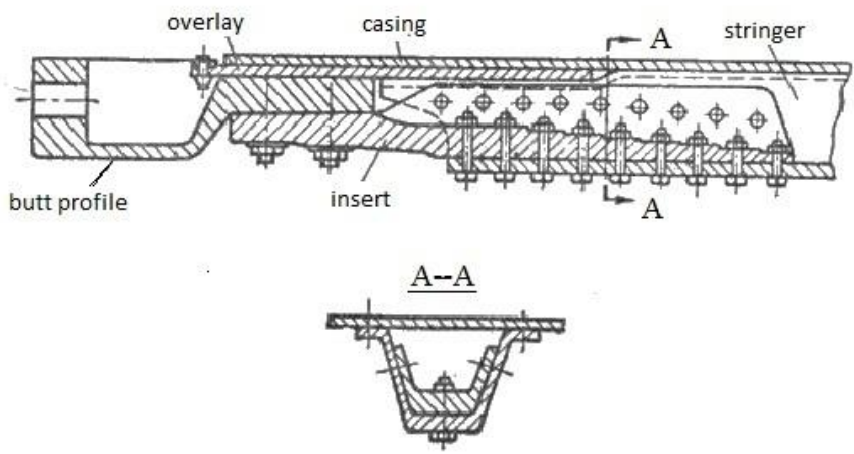

Fig. 3. The design of the node that connects the butt profile with the plating and stringers.

All methods of measuring of linear and angular dimensions can be divided into contact and contactless. The primary converter using contact method is in direct contact with the object, for example, with help of the probe. However, using the same instruments for measuring of strain in dynamic testing is impractical, as there can be quite large error due to lead probe from the controlled surface.

Preferred contact means for strain measuring uses the resistive-strain sensors that are placed in the area of the alleged critical strain. But the range of strain measured by the resistivestrain sensors is very limited, training and consolidation of sensors rather require painstaking and time-consuming, rigid mountings are unreliable for extended use, you need to provide compensation of temperature instability for measurements, the shock loads of resistive-strain sensors can not withstand even within the permissible deformation. Therefore, the resistivestrain sensors are mainly used for static tests.

In the non-contact method of measuring the primary transducer is not directly in contact with the object and does not distort its parameters. According to physical laws underlying in base of the principle of action, the contactless transducers of non-electrical quantities into electrical quantities can be divided into inductive, capacitive, optical and ultrasonic [2], [3].

According to physical laws underlying in base of the principle of action, the contactless transducers of nonelectrical quantities into an electrical quantities can be divided into inductive, capacitive, optical and ultrasonic [2], [3]. Analysis of the devices, that implement these methods, shows that measuring deformation by contactless method is more appropriate to use capacitive sensors.

Capacitive sensors are based on the conversion of the input variable into variable capacitance. Common capacitive sensors that transform the measured geometrical parameters into capacity due to changes in the gap between the plates (electrodes) of the capacitor. But the measure of deformation structures can only be one-sided approach to the controlled surface. In addition, the sensor capacitance value through design miniature of probe sensor is very small, making it difficult to measure. These circumstances make such design of the capacitive sensor and measuring circuit, which would be suitable for problem decision to be considered. 
The closest the technical solution is micro-movement meter designed to control the forming of structure during electro-welding [4]. The device was designed to measure the micro-movement of checkpoint arising from thermal deformation of sheet metal during welding. The sensitive element of the device (probe) is a differential capacitive transducer with the "open" field (Fig. 4). The range of measurable movement was $1.2 \mathrm{~mm}$, the error of measurement is less than $0.003 \mathrm{~mm}$. Displacement can be measured by two coordinates alternately. The design of the probe has a round body, which includes a system of electrodes of capacitive transducer that generates an electromagnetic field. Electrodes $1, l^{\prime}$ i $l^{\prime \prime}, l^{\prime \prime \prime}$ (the last two in the figure are not marked) are high-power electrodes arranged in pairs in mutually perpendicular planes, 2 is low-power electrode. "Mask" of 3 probe with body 4 and the copper tube-screens 5 are assigned to avoid parasitic capacitances of connecting conductors of sensor. The probe of sensor is placed on the "control point", which is a metal cylinder, diameter of which is equal to the width of the low-potential electrode 2 and height is $1 \mathrm{~mm}$, with a thin cylindrical base, which is attached to the surface. Electrode system allows for the various options of connectivity of electrodes to measuring system to obtain more information about the current strain, including the direction and magnitude of micro-movement on the plane of controllable surface, and (if relevant connection) about distance to the controlled surface, it is possible to measure the movement of the three coordinates alternately [4]. The principle of operation of the reporting unit can be used to create a device for continuous deformation control of structural elements of the aircraft.

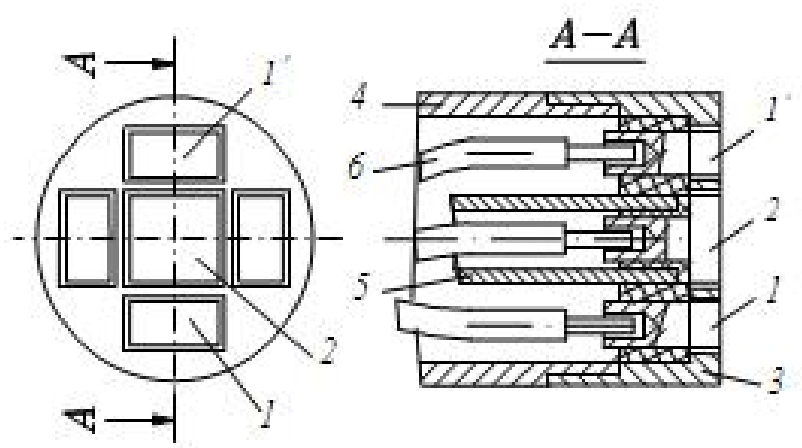

Fig. 4. Probe of capacitive sensor of micro-movement: $1, l^{\prime}$ are high-power electrodes; 2 is the low-power electrode; 3 is the "Mask" of probe; 4 is the building; 5 is the screen of low power electrode.

Considering the above, the design options of sensitive element were developed. The design of the sensitive element for deformation sensor of butt profile and its location is shown in Fig. 5

Given that the most likely occurrence of plastic deformation in the area of bolted joints, it is advisable to place a similar device on the ends of bolts of this design, one of these ends can be used as a "reference point". The use of the proposed device allows for a "control point" to use head rivets, performance design and other geometric designs of heterogeneity of structural elements of the aircraft. If the measured strain has the famous character of changes, it is possible to simplify the design of the probe to measure one coordinate. The design of the sensor has one a feature that is associated with micro-measurement values of capacitance.

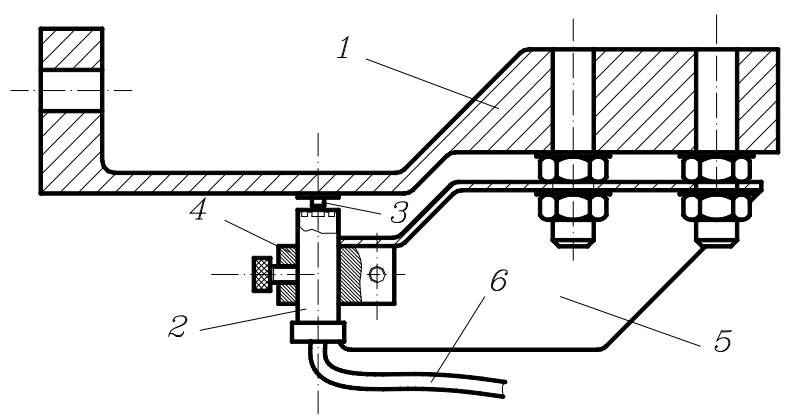

Fig. 5. Placement of sensitive element of the sensor (probe) for control of the deformation of butt profile: 1 is the butt profile; 2 is the probe; 3 is the control point; 4 is the clamp of probe; 5 is the tacking fashioned box; 6 is the connectors

The connecting lines must be strictly shielded, so they are placed in the appropriate copper tubes of small diameter.

There will be a set of sensors considered the final stage of drawing up the aircraft or during repair.

Developed critical deformation monitoring system elements of aircraft design is shown in Fig. 6.

The system consists of a set of sensors micromovings 1, 2, $3, \ldots, N$, located on the controllable elements of aircraft construction, power generator bridge, shoulder transformer, switch to switch position measurement, on-board computer (Beom) or part thereof for a separate the system processor and the LED status monitoring. Beom should have several (number of controlled areas) multi-channel analog-to-digital converters ADC. The device is non-volatile memory is loaded processing program, operation of the system software and a database of valid values of deformations in controlled locations. Modes of operation of the system asks the operator: Enables control of all areas, the choice of a particular area or even a specific sensor with the specified direction of deformation monitoring. The information comes in the light, where the LA circuit vividly displayed in terms of dangerous condition of the structure. The value of current and permissible deformation at the point requested by the operator is displayed at the bottom of the display.

\section{CONCLUSION}

The implementation of the system for detection of critical deformation elements of aircraft construction is proposed. The structure of sensitive element sensor is considered. The measuring system will ensure continuous monitoring of the state of the aircraft construction.

The structure of digital system for the detection of critical deformation elements of aircraft construction is considered. 


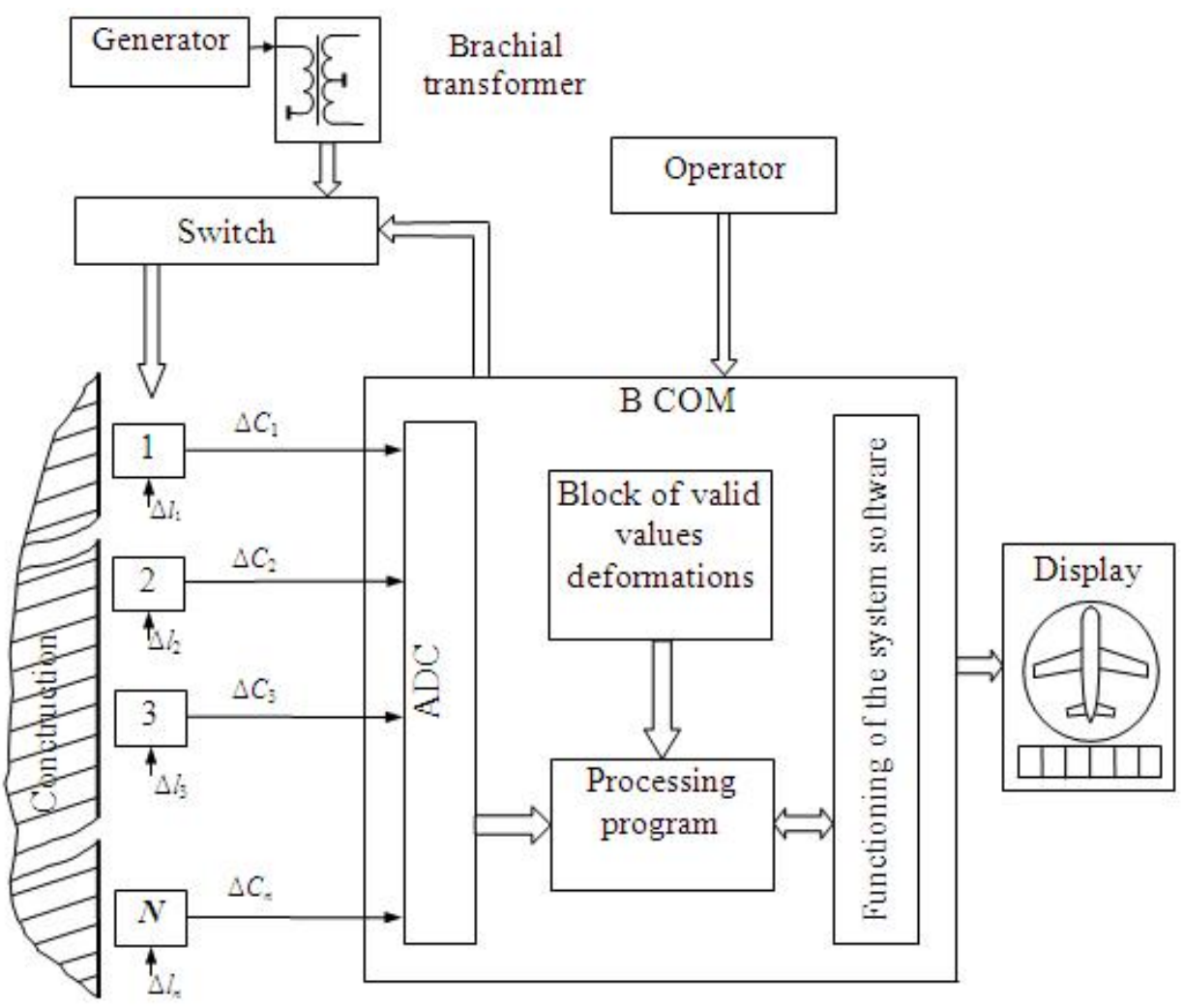

Fig. 6. System of detection of critical deformation elements of aircraft construction.

\section{REFERENCES}

[1] V.L. Belsky, J. P. Vlasov, N.V. Zaitsev et al. Construction of aircraft. Moscow, Oborongiz, 1965, $710 \mathrm{p}$.

[2] S.A. Spector. Electrical measurements physical quantities, measurement methods. Leningrad, Energoatomisdat, 1987, 320 p.
[3] F.B. Grinevich and A.I. Novik. Test Compensatory and bridge Devices capacitive sensors. Kiev, Naukova Dumka. 1987, 112 p.

[4] A.K. Ablesimov, A.Z. Gaydamaka, and A.P. Kozlov. "Non-contact measurement of the micromovings." Elektroniki and the control system, Kyiv, NAU, vol. 3(5), 2005, pp. 141-146. 